EPJ Web of Conferences 59, 17016 (2013)

DOI: $10.1051 /$ epjconf/20135917016

(C) Owned by the authors, published by EDP Sciences, 2013

\title{
Suppression effects of Weibel instability for fast electron divergence
}

\author{
H. Sakagami ${ }^{1, a}$, T. Johzaki ${ }^{2}$, T. Taguchi ${ }^{3}$ and K. Mima ${ }^{4}$ \\ ${ }^{1}$ Fundamental Physics Simulation Div., National Inst. for Fusion Science, Toki 509-5292, \\ Japan \\ ${ }^{2}$ Graduate school of Engineering, Hiroshima University, Higashihiroshima 739-8511, Japan \\ ${ }^{3}$ Dept. of Electrical and Electronic Eng., Setsunan University, Neyagawa 572-8508, Japan \\ ${ }^{4}$ Institute of Laser Engineering, Suita 565-0871, Japan
}

\begin{abstract}
Quasi-static magnetic fields, which are induced by the Weibel instability and grow to more than hundred Megagauss, lead to large divergence angle of fast electrons, hence lower energy coupling. To suppress the divergence, two different structures, namely density trough and punched out holes, are introduced to targets. In the density trough target, the Weibel instability is enhanced and the divergence is getting worse. On the other hand, the divergence angle is improved but the number of electrons is degraded for fast electrons $(<3 \mathrm{MeV})$ in the punched out target.
\end{abstract}

\section{INTRODUCTION}

In the fast ignition (FI) scheme, a fuel target is imploded by long-pulse implosion lasers and its compressed core is heated by a short-pulse ultrahigh-intense laser. The FIREX-I (Fast Ignition Realization Experiment phase-I) project aims to demonstrate that the compressed core could be heated up to $5 \mathrm{keV}$ using gold $\mathrm{(Au}$ ) cone-guided targets, and incorporated FIREX-I experiments have started at ILE, Osaka University. Efficient heating mechanisms and achievement of such high temperature have not been, however, clarified yet, and we have been promoting the Fast Ignition Integrated Interconnecting code $\left(\mathrm{FI}^{3}\right)$ project to boldly explore FI frontiers [1-3]. First series of the incorporated experiments were performed in 2009, and only 30 -fold enhancement in neutron yield, which was $\sim 1 / 30$ smaller than that in 2002 experiments, was achieved and lower energy coupling from the heating laser to the imploded core was anticipated [4].

According to 2D PIC simulations, it is pointed out that fast electrons, which are generated by interactions between the heating laser and the Au cone tip, have a large divergence angle ( $~ 90$ degree), and a small number of them can hit the core for heating even if preformed plasmas inside the core have a small density scale length [4]. The Weibel instability [5, 6], which is induced by a fast electron stream and its counter stream of background electrons, generates a quasi-static magnetic field, and it grows to more than hundred Megagauss. This field is large enough to scatter several MeV electrons within $1 \mu \mathrm{m}$, and these divergent fast electrons result in low coupling efficiency.

According to the linear dispersion relation of the Weibel instability, the growth rate depends on the temperature of background electrons and it grows more slowly with higher temperature [9]. It is confirmed that higher background temperature leads to slower growth of the Weibel instability and smaller divergence angle of fast electrons by 2D PIC simulations. For the next step, the specific target

\footnotetext{
ae-mail: sakagami.hitoshi@nifs.ac.jp
}

This is an Open Access article distributed under the terms of the Creative Commons Attribution License 2.0, which permits unrestricted use, distribution, and reproduction in any medium, provided the original work is properly cited. 


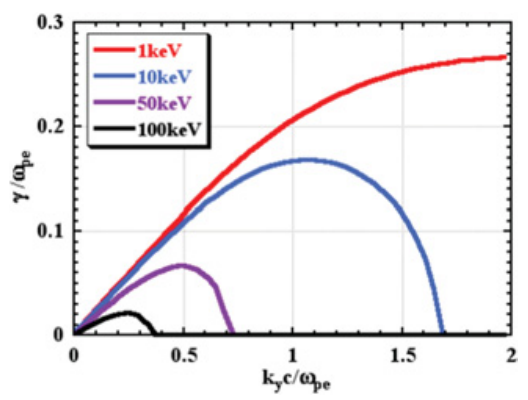

Figure 1. The linear growth rates of the Weibel instability.

structure is introduced to raise the temperature of background electrons by the fast electron flow and suppression effects of the Weibel instability for fast electron divergence is evaluated.

\section{WEIBEL MAGNETIC FIELD AND FAST ELECTRON DIVERGENCE}

The Weibel instability is invoked by fast electron streams produced by the laser pulse and return flows carried by background electrons, which are induced to maintain current neutrality. These oppositely directed currents repel each other, making the distribution of the current density in the transverse direction. It generates large quasi-stationary magnetic fields transverse to the direction of fast electron stream, scattering fast electrons with large angles. As various linear electromagnetic instabilities are developed under such electron current condition, sophisticated analyses are perfomed in Refs. [7, 8]. But assumptions of cold or warm plasmas in those Refs. are not suitable for our FI condition, and we adopt the linear analysis of Refs. [9, 10]. The linear growth rates of the Weibel instability for different background electron temperatures are shown in Figure 1 for $n_{\text {fast }}: \mathrm{n}_{\text {back }}=1: 9, \mathrm{~T}_{\text {fast }}=100 \mathrm{keV}$ and $\gamma_{\text {fast }}=9.5$. Higher temperature leads to lower growth rate and longer wavelength that gives maximum growth.

To check this characteristic depending on the background electron temperature, the Weibel instability is investigated with the use of 2D PIC code. In simulations, the $\mathrm{Au}(\mathrm{A}=197, \mathrm{Z}=30$ ) cone tip is introduced as a $10 \mu \mathrm{m}$ thickness, $6( \pm 3) \mu \mathrm{m}$ wide, $20 \mathrm{n}_{\mathrm{cr}}$ flat profile with a preformed plasma, which has a exponential profile of the scale length $\left(\mathrm{L}_{\mathrm{pre}}=0.5 \mu \mathrm{m}\right)$ with density from 0.1 up to $20 \mathrm{n}_{\mathrm{cr}}$. Initial temperature of electrons is set to 1,10 or $100 \mathrm{keV}$, and immobile ions are used to ignore surface deformation effects. The heating laser is set to $\lambda \square=1.06 \mu \mathrm{m}, \mathrm{I}_{\mathrm{L}}=10^{20} \mathrm{~W} / \mathrm{cm}^{2}, \tau_{\text {rise }}=5 \mathrm{fs}, \tau_{\text {flat }}=\infty$ as a plane wave. Periodic boundary conditions are employed for both particles and fields in transverse direction, and fast electrons are observed at $4.5 \mu \mathrm{m}$ from the right plasma edge. To ignore a circulation of fast electrons, we introduce an artificial cooling region $(1 \mu \mathrm{m}$ width), in which fast electrons are gradually cooled down to the initial temperature, behind the observation point. Quasi-static transverse magnetic fields $\left(B_{z}\right)$ at 50 fs are shown in Figure 2 for different background electron temperatures $\left(T_{\text {back }}\right)$ (a) 1 , (b) 10 and (c) $100 \mathrm{keV}$. In the $\mathrm{T}_{\text {back }}=1$ or $10 \mathrm{keV}$ cases, $\mathrm{B}_{\mathrm{z}}$ grows up to $\pm 100 \mathrm{MG}$ and almost saturates around that value near the laser-plasma interaction region where fast electron streams are produced, and fast electrons are scattered by $B_{z}$. On the other hand, large $B_{z}$ is not generated at that region due to the low growth rate and fast electrons can flow deeply inside the plasma and a longitudinal structure of $B_{z}$ is generated in the case of $T_{\text {back }}=100 \mathrm{keV}$.

Time integrated $(0 \sim 100 \mathrm{fs})$ angular distributions of forward fast electrons are shown in Figure 3 for (a) $\mathrm{T}_{\text {back }}=1$, (b) 10 and (c) $100 \mathrm{keV}$. As $\mathrm{B}_{\mathrm{z}}$ quickly grows and saturates, there is no clear difference in fast electron divergence between the cases of $\mathrm{T}_{\text {back }}=1$ and $10 \mathrm{keV}$. However, the divergence in the case of $\mathrm{T}_{\text {back }}=100 \mathrm{keV}$ is smaller than that of the other cases. According to the conserved transverse canonical momentum in a simple $1 \mathrm{D}$ framework, higher $\mathrm{T}_{\text {back }}$ leads to more isotropic fast electrons. 

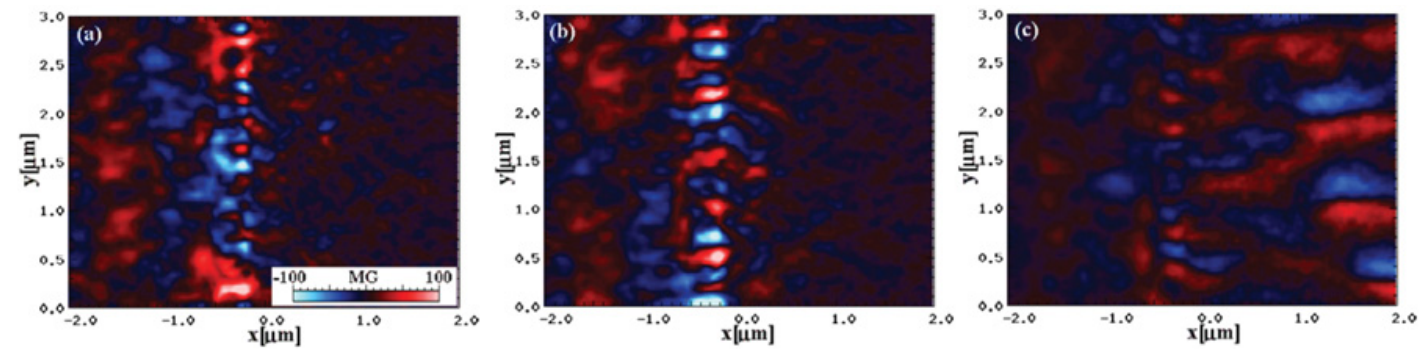

Figure 2. Quasi-static transverse magnetic field profiles at $50 \mathrm{fs}$ for (a) $\mathrm{T}_{\text {back }}=1$, (b) 10 and (c) $100 \mathrm{keV}$.
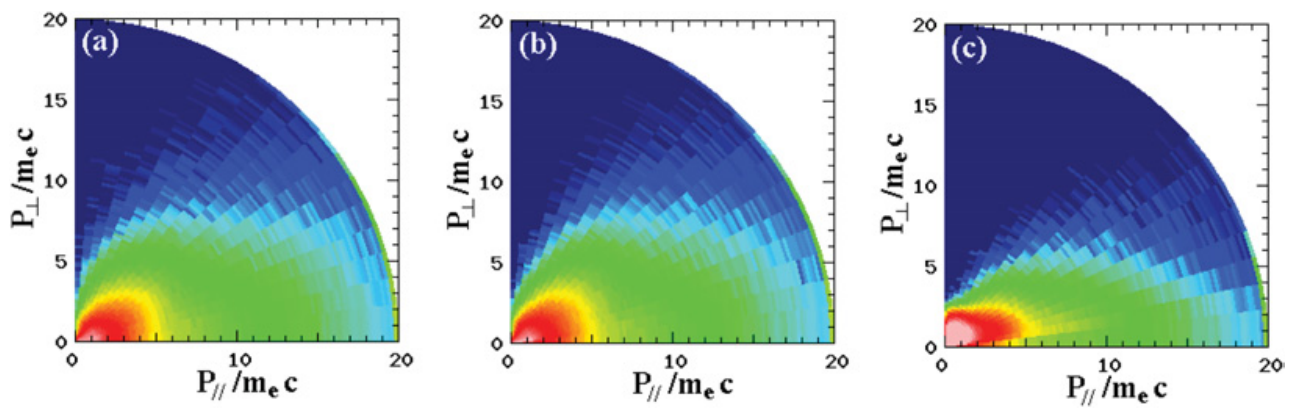

Figure 3. Time integrated angular distributions of forward fast electrons for (a) $\mathrm{T}_{\text {back }}=1$, (b) 10 and (c) $100 \mathrm{keV}$.

As even highest $T_{\text {back }}(100 \mathrm{keV})$ is much less than the fast electron energy $(>1 \mathrm{MeV})$, this effect cannot be seen in Figure 3. If a specific target structure can be introduced to raise the temperature of background electrons by any kind of fluid instabilities, suppression effects of the Weibel instability for fast electron divergence may be expected.

\section{STRUCTURED TARGETS}

\subsection{Density trough targets}

Background electrons must flow to neutralize the fast electron current, forming the return current. If a density trough against the flow direction exists, the flow speed of background electrons increases according to decrement of background electron density. This stream excites a strong two-stream instability, in which background electrons are heated up [1]. Therefore, we introduce the density trough, which is $10 \mathrm{n}_{\mathrm{cr}}$ and $0.5 \mu \mathrm{m}$ thickness, into the target as shown in Figure 4(a) and the other parameters are same as previous simulations. $\mathrm{B}_{\mathrm{z}}$ at $50 \mathrm{fs}$ for $\mathrm{T}_{\text {back }}=1 \mathrm{keV}$ is shown in Figure 5(a). It is noted that the plot range in Figure 2 is $\pm 100 \mathrm{MG}$, but $\pm 300 \mathrm{MG}$ in Figure 5 . With this target, it is observed that background electrons are heated up to more than $100 \mathrm{keV}$ by the two-stream instability and the scale length of magnetic structures becomes larger than that of without trough case. The Weibel instability is, however, enhanced by the strong flow of background electrons, leading to large $\mathrm{B}_{\mathrm{z}}$ around $200 \mathrm{MG}$, twice larger than that in Figure 2(a). As a result, fast electron divergence is getting worse and this structure does not work well.

\subsection{Punched out targets}

As the growth rate of the Weibel instability is absolutely large, little suppression effects can be expected even if the background electron temperature increases to more than $100 \mathrm{keV}$. $\mathrm{B}_{\mathrm{z}}$ grows up to $\pm 100 \mathrm{MG}$ 

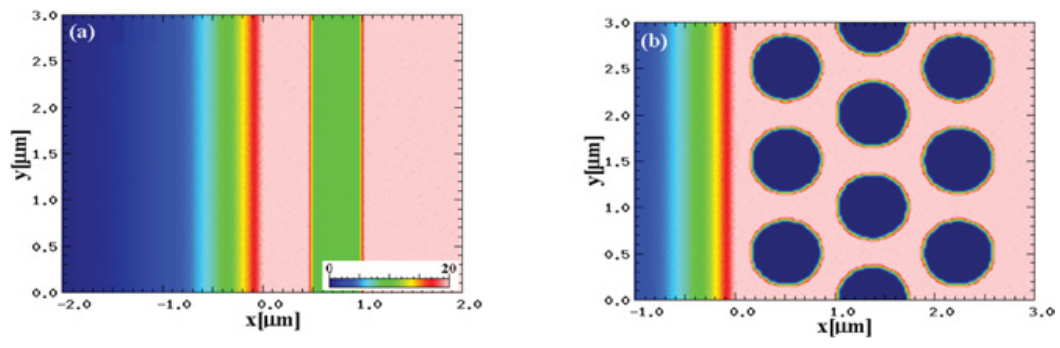

Figure 4. Electron density profiles for (a) density trough target and (b) punched target.
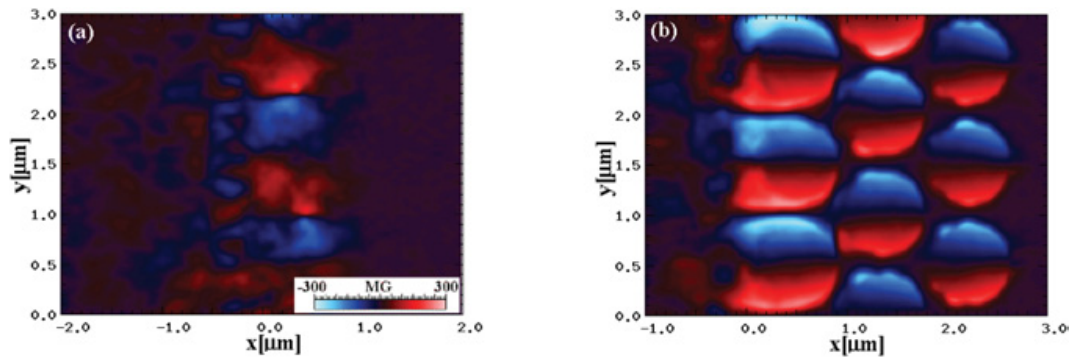

Figure 5. Quasi-static transverse magnetic field profiles at $50 \mathrm{fs}$ for (a) density trough target (b) punched target.
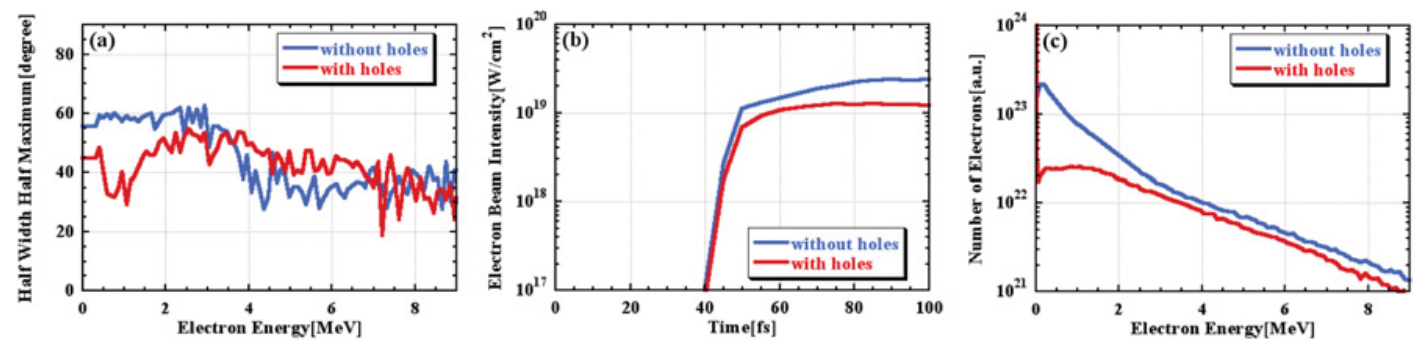

Figure 6. Fast electron characteristics for without or with punched out holes: (a) half width half maximum of divergence at $100 \mathrm{fs}$, (b) time evolutions of fast electron beam intensity and (c) time integrated fast electron energy distribution.

sooner or later, scattering fast electrons. This magnetic field does not remain at rest and shows turbulent behaviors, enhancing the divergence of fast electrons. To control the location of $\mathrm{B}_{\mathrm{z}}$, punched out targets are introduced, where many vacuum holes are punched out (diameter is $0.7 \mu \mathrm{m}$, distance between each hole center is $1 \mu \mathrm{m}$ ) as shown in Figure 4(b), and the other parameters are same as previous simulations. $\mathrm{B}_{\mathrm{z}}$ at $50 \mathrm{fs}$ for $\mathrm{T}_{\text {back }}=1 \mathrm{keV}$ is shown in Figure 5(b). Background electrons that carry the return current can only flow in the gaps between holes, because the sheath field prevents them from crossing over the hole. Thus stable magnetic fields are induced and grow up to almost $\pm 300 \mathrm{MG}$. Fast electrons go forward with a meandering motion, and reduction of divergence can be expected. Half width half maximum of divergence at $100 \mathrm{fs}$, time evolutions of fast electron beam intensity and time integrated fast electron energy distribution without or with punched out holes are shown in Figure 6(a), (b) and (c), respectively. In the case with punched out holes, divergence angle decreases when fast electron energy is less than $3 \mathrm{MeV}$. Unfortunately, this structure simultaneously disturbs fast electron propagation, and the fast electron beam intensity also decreases because the number of electrons in such energy range is reduced. 


\section{IFSA 2011}

In the punched out target, the divergence angle is improved but the number of electrons is degraded for fast electrons $(<3 \mathrm{MeV})$ which can efficiently heat the core. Therefore we will perform integrated simulations by $\mathrm{FI}^{3}$ to evaluate the core heating performance of this target structure.

This work is partially supported by JSPS Grant-in-Aid for Scientific Research (C)(22540511).

\section{References}

[1] H. Sakagami, T. Johzaki, H. Nagatomo, K. Mima, Laser Part. Beams 24, 191 (2006)

[2] T. Johzaki, H. Sakagami, H. Nagatomo, K. Mima, Laser Part. Beams 25, 621 (2007)

[3] H. Sakagami, T. Johzaki, H. Nagatomo, K. Mima, Nucl. Fusion 49, 075026 (2009)

[4] T. Johzaki, et al.,Nucl. Fusion 51, 073022 (2011)

[5] E. S. Weibel, Phys. Rev. Lett. 2, 83 (1959)

[6] F. Califano, F. Pegoraro, S. V. Bulanov, Phys. Rev. E 56, 963 (1997)

[7] A. Bret, M.-C. Firpo, C. Deutsch, Phys. Rev. E 72, 016403 (2005)

[8] B. Hao, et al., Phys. Rev. E 80, 066402 (2009)

[9] Y. Sentoku, K. Mima, S. Kojima, H. Ruhl, Phys. Plasmas 7, 689 (2000)

[10] T. Taguchi, T. M. Antonsen Jr., C. S. Liu, K. Mima, Phys. Rev. Lett. 86, 5055 (2001) 